\title{
New Developments in Subsurface Flow and Transport
}

\author{
J. Jaime Gómez-Hernández
}

Received: 17 January 2012 / Accepted: 18 January 2012 / Published online: 26 January 2012

(C) International Association for Mathematical Geosciences 2012

This special issue of Mathematical Geosciences is a good showcase of some of the currently active fronts of research on flow and transport in the subsurface. The six papers included address issues related to aquifer and reservoir characterization, flow and transport in both saturated and unsaturated zones, complex modeling of multiple transport processes, inverse modeling, channeling and connectivity.

In the first paper, Fiori et al. focus on the characterization of high velocity channels in heterogeneous aquifers. They show that the larger the conductivity variance, the more noticeable these channels become, and that their length is always larger than the conductivity integral scale. An important conclusion of this paper is that connectivity is always stronger on three-dimensional models than on two-dimensional ones, therefore alerting any practitioner against using two-dimensional codes to model any process, such as contaminant transport, in which the connectivity of high velocity zones is significant.

The second paper, by Cherpeau et al., also touches upon the issue of connectivity, although in this case, it is related to fault connectivity in a discrete fracture network (DFN) model, as part of a larger effort for the characterization of faults at multiple scales. This paper presents an inverse modeling approach to condition a DFN model to dynamic data in which the parameters of the inversion are the structural data defining the number, position and orientation of the fractures. This is contrary to more standard approaches that invert the flow parameters, such as conductivity or porosity, directly.

The third paper, by Zhou et al., also deals with how to construct a geologically plausible model through inverse modeling. In this case, the use of multiple-point geostatistics together with a modified ensemble Kalman filter approach is capable of

J.J. Gómez-Hernández $(\bowtie)$

Universitat Politècnica de València, Camino de Vera s/n, 46022 Valencia, Spain

e-mail: jgomez@upv.es 
generating conductivity realizations that honor both the channeled geology extracted from a training image and the dynamic data measured in a number of piezometers. An important detail about this paper is that no conductivity data are used, concluding that the prior model (given by the training image) plus the piezometric head data suffice to obtain a good characterization of the aquifer.

The emphasis of the next three papers is on transport. The fourth paper, by Jiménez-Martínez et al., presents a rather complex multiphase model in the unsaturated zone, illustrated with an experiment showing how tritium is naturally attenuated after a point injection into the upper soil layers. Tritium evolution is largely affected by evapotranspiration and tritium re-emission into the atmosphere.

The fifth paper, by Sena et al., goes one step further in the complexity of transport modeling by including reactive transport and microbial processes. Microbes are ubiquitous in groundwater and they may affect the fate of dissolved organic contaminants. The paper revisits the mathematics of these processes and then performs a comparison of two available computer codes to model the aerobic benzene degradation in a shallow and oxidizing aquifer.

Finally, the sixth paper, by Mejía et al., gives a very clear message on the importance of properly sealing abandoned wells and of properly screening active wells in multi-aquifer systems in order to prevent the contamination by nitrate of the deep aquifers in these systems. Nitrate contamination is a significant problem in Mediterranean aquifers, and the common practice is to first move the pumps from the upper aquifers to the deeper ones, and later to abandon the wells. If these wells are not properly handled, they become pathways for nitrate migration between aquifers. The paper also alerts readers to the extremely biased image of an aquifer's chemical state that a simple interpolation (as is commonly done) of point concentration measurements provides.

I wish to express my deep appreciation to Roussos Dimitrakopoulos, Editor-inChief of Mathematical Geosciences, for allowing me to handle this special issue, and for his help and support while assembling it.

There are other more specialized journals dealing with groundwater research, but there is no doubt that flow and transport in the subsurface is an important member of mathematics in the geosciences. 